\title{
Recurrence patterns and risk factors following thoracoscopic esophagectomy with radical lymph node dissection for thoracic esophageal squamous cell carcinoma
}

\author{
ITASU NINOMIYA $^{1}$, KOICHI OKAMOTO ${ }^{1}$, TOMOYA TSUKADA ${ }^{1}$, JUN KINOSHITA ${ }^{1}$, \\ KATSUNOBU OYAMA $^{1}$, SACHIO FUSHIDA ${ }^{1}$, HARUSHI OSUGI ${ }^{2}$ and TETSUO OHTA ${ }^{1}$ \\ ${ }^{1}$ Gastroenterologic Surgery, Department of Oncology, Division of Cancer Medicine, Graduate School of Medical Science, \\ Kanazawa University, Kanazawa, Ishikawa 920-8641; ${ }^{2}$ Department of Gastroenterological Surgery, \\ Osaka City University, Graduate School of Medicine, Osaka 565-0871, Japan
}

Received April 9, 2015; Accepted October 19, 2015

DOI: $10.3892 / \mathrm{mco} .2015 .688$

\begin{abstract}
The aim of the present study was to clarify the therapeutic effect of thoracoscopic esophagectomy with radical lymph node dissection based on the recurrence pattern, and identify the risk factors for relapse-free survival in patients with esophageal cancer. The recurrence patterns in 140 patients who underwent complete thoracoscopic radical esophagectomy between January 2003 and December 2012 were investigated. The risk factors for recurrence were examined by univariate and multivariate analysis. Mediastinal recurrence in association with initial lymphatic metastasis was precisely analyzed. Esophageal cancer recurred in $49(35.0 \%)$ of the 140 patients. The median recurrence time was 259 (45-2,560) days after the initial treatment. The patterns of initial recurrence among the 140 patients included hematological recurrence in 24 patients (17.1\%), lymphatic recurrence in $26(18.6 \%)$, pleural dissemination in $5(3.6 \%)$, peritoneal dissemination in $2(1.4 \%)$, and local recurrence in $4(2.9 \%)$. Lymphatic recurrence within the mediastinal regional lymphatic stations occurred in only 8 (5.7\%) of the 140 patients. Univariate analysis for relapse-free survival showed that the statistically significant variables were a tumor location in the upper third of the esophagus, stage of pT3 or pT4, presence of nodal metastasis, pStage of III or IV, presence of a residual tumor, performance of preoperative chemotherapy and performance of postoperative therapy. Multivariate analysis showed that only nodal metastasis and a positive residual tumor were statistically significant independent risk factors for relapse-free survival. Lymphatic recurrence within the mediastinum, particularly the station around the bilateral recurrent
\end{abstract}

Correspondence to: Dr Itasu Ninomiya, Gastroenterologic Surgery, Department of Oncology, Division of Cancer Medicine, Graduate School of Medical Science, Kanazawa University, Takaramachi 13-1, Kanazawa, Ishikawa 920-8641, Japan

E-mail: nino@staff.kanazawa-u.ac.jp

Key words: recurrence, risk factors, esophageal cancer, squamous cell carcinoma, thoracoscopy laryngeal nerves, was infrequent and independent of the initial metastatic distribution. Thoracoscopic esophagectomy with radical lymph node dissection provides favorable locoregional control. Lymphatic recurrence within the mediastinal regional nodes is infrequent and independent of the initial lymph node metastasis. A pathological residual tumor and lymph node metastasis are significant risk factors for recurrence.

\section{Introduction}

Surgical resection is the primary therapy for local and locoregional disease in patients with esophageal cancer. The optimal extent of resection of the esophagus and regional nodes remains under dispute. Radical or en-bloc esophagectomy aims to remove the primary cancer with extensive systematic lymph node dissection involving radical dissection of the upper abdominal, mediastinal and lower cervical nodes (three-field dissection). This aggressive surgery with three-field lymph node dissection is reportedly associated with longer patient survival compared to the less extensive thoracoabdominal (two-field) dissection $(1,2)$. Udagawa et al (3) reported the effectiveness and importance of wide-range lymph node dissection in patients with thoracic esophageal cancer by investigating the effectiveness of lymphadenectomy.

Despite undergoing radical surgery, 42.5 to $52.4 \%$ of patients develop recurrence, and the prognosis of these patients remains poor (4-7). A greater depth of invasion and/or lymph node metastasis extension are previously reported risk factors for recurrence $(3,8,9)$.

Video-assisted thoracoscopic (VATS) radical esophagectomy (VATS-esophagectomy) has been developed to reduce the severity of surgical insult in the management of esophageal cancer (10-12). Furthermore, certain retrospective studies have shown that the oncological effectiveness of thoracoscopic surgery is comparable to that of open thoracotomy (13-15). VATS-esophagectomy also provides adequate locoregional control of VATS-esophagectomy comparable to that of open surgery (14).

Our previous study reported the introduction of VATS-esophagectomy in the left lateral position for the 
treatment of esophageal cancer in January 2003 (16). Our primary objective was to introduce VATS-esophagectomy and confirm that it was safe, and monitor the exhaustive lymph node dissection under the magnifying effect of a videoscope. Following the induction period, the indications for VATS-esophagectomy were expanded to include advanced esophageal cancer as the main therapeutic strategy in multimodality therapy. The long-term oncological outcomes following VATS-esophagectomy were favorable if curative surgery could be performed (17). However, the overall oncological therapeutic effect of VATS-esophagectomy remains unclear.

The present study aimed to clarify the therapeutic effect of VATS-esophagectomy by evaluating the recurrence patterns of thoracic esophageal squamous cell carcinoma in patients who underwent VATS-esophagectomy. In particular, the lymphatic recurrence patterns were investigated to show the effect of mediastinal dissection during thoracoscopic surgery. Finally, the risk factors for relapse-free survival were assessed.

\section{Materials and methods}

Patients. Data were obtained from 140 patients who underwent complete VATS-esophagectomy for thoracic esophageal squamous cell carcinoma at Kanazawa University Hospital (Kanazawa, Japan) between January 2003 and December 2012. The data were retrospectively collected and analyzed, and all the patients were followed until fatality or December 2014 (i.e., at least 2 years after surgery). The criteria for VATS-esophagectomy included no previous radiation therapy; pulmonary function capable of sustaining single-lung ventilation; no concomitant serious medical conditions such as liver cirrhosis, heart failure or renal failure; and patient preference for VATS-esophagectomy. VATS-esophagectomy was performed for T4 tumors that were considered technically resectable after induction chemotherapy from August 2008. Clinicopathological characteristics (tumor invasion, node, metastasis and stage) were based on the tumor-node-metastasis (TNM) classification (7th edition), by the International Union Against Cancer (18). Lymph node station spread was determined according to the Japanese classification system $(19,20)$.

Surgical procedure. All the patients underwent esophagectomy and reconstruction, as previously described (16). During the thoracic procedure, patients were placed in the left lateral position. Thoracoscopic esophagectomy with mediastinal lymph node dissection was performed from a $5-\mathrm{cm}$ minithoracotomy and four 12-mm trocars or six ports without minithoracotomy. The right lung was deflated, and the mediastinal lymph nodes were carefully dissected. Dissection of the lymph nodes around the bilateral recurrent laryngeal nerves was performed with caution (17). Abdominal and supraclavicular cervical lymph node dissection was performed simultaneously with the patient in the supine position. The gastric tube was selected as the primary reconstruction conduit. The surgery was finished with cervical anastomosis. Cervical lymph node dissection was omitted in patients with a T1 tumor in the lower thoracic esophagus, serious medical conditions or suspicion of a residual tumor.

In cases of noncurative resection, the retrosternal route was selected for elevation of the gastric tube to allow for subsequent chemoradiotherapy of the residual tumor. The small intestinal conduit was used in patients who had previously undergone gastrectomy or who required total gastrectomy for treatment of simultaneous gastric cancer.

Neoadjuvant or adjuvant treatment. All the patients with resectable tumors initially underwent VATS-esophagectomy. Patients with pathologically positive lymph node metastasis subsequently underwent adjuvant chemotherapy using a combination of cisplatin and 5-fluorouracil. From January 2008, neoadjuvant chemotherapy comprising of cisplatin and 5-fluorouracil was performed for stage II and III tumors. From February 2012, the neoadjuvant chemotherapy protocol was changed to a combination of docetaxel, cisplatin and 5-fluorouracil for stage III and IV tumors. Patients with pathologically positive radial margins underwent postoperative chemoradiotherapy.

Follow-up. All the patients were followed up at the outpatient department at Kanazawa University Hospital. Routine follow-up examinations included measurement of serum tumor markers and performance of a CT scan from the neck to the upper abdomen at a 6-month interval for 5 years, and following this, annually. Endoscopy was performed annually. Diagnosis of recurrence was based on histological, cytological or radiological evidence. The interval from the first treatment to the detection of recurrence was defined as the time to recurrence.

Data analysis. The $\chi^{2}$, Fisher's exact and Student's t-tests were used to compare the categorical variables. Relapse-free survival curves according to the TNM classification were estimated by the Kaplan-Meier method. McNemar's test was used to compare the difference in the metastasis distribution in each lymphatic station between the primary surgery and the initial recurrence. Univariate and multivariate analyses by means of Cox regression models were performed to assess the prognostic factors for relapse-free survival. The full-model approach incorporating all candidate variables was used in the multivariate analysis to avoid overfitting and selection bias. $\mathrm{P}<0.05$ was considered to indicate a statistically significant difference. All the analyses were performed with IBM SPSS Statistics for Windows, version 22.0 (IBM Corp., Armonk, NY, USA).

\section{Results}

Patient demographics and recurrence pattern. Esophageal cancer recurred in $49(35.0 \%)$ of the 140 patients. The clinicopathological characteristics of the patients with and without recurrence are shown in Table I. Among the various factors, the tumor location, histological grade $(\mathrm{G})$, depth of invasion (pT), number of lymph node metastases $(\mathrm{pN})$, tumor stage (pStage), presence of a residual tumor $(\mathrm{R})$, preoperative chemotherapy and adjuvant therapy were associated with the occurrence of recurrent disease. The median recurrence time was $259(45-2,560)$ days after the initial treatment. A total of 34 (69.3\%) and $45(91.8 \%)$ of the 49 patients with recurrence showed recurrence within 1 and 2 years, respectively (Fig. 1). Additionally, all the patients except one developed recurrence within 3 years.

Among all 140 patients, the patterns of initial recurrence included hematological recurrence in 24 patients $(17.1 \%)$, lymphatic recurrence in $26(18.6 \%)$, pleural dissemination in 
Table I. Clinicopathological characteristics of patients with and without recurrence.

\begin{tabular}{|c|c|c|c|c|}
\hline \multirow[b]{2}{*}{ Variables } & \multirow[b]{2}{*}{ Total } & \multicolumn{2}{|c|}{ Recurrence, n (\%) } & \multirow[b]{2}{*}{ P-value } \\
\hline & & Negative & Positive & \\
\hline Case & 140 & $91(65.0)$ & $49(35.0)$ & \\
\hline Age, mean years \pm SD & $66.1 \pm 7.5$ & $66.2 \pm 7.0$ & $65.7 \pm 8.4$ & $0.763^{\mathrm{a}}$ \\
\hline Gender & & & & $0.298^{b}$ \\
\hline Male & 115 & $77(67.0)$ & $38(33.0)$ & \\
\hline Female & 25 & $14(56.0)$ & $11(44.0)$ & \\
\hline Location & & & & $0.084^{\mathrm{b}}$ \\
\hline Upper third & 22 & $10(45.5)$ & $12(54.5)$ & \\
\hline Middle third & 77 & $51(66.2)$ & $26(33.7)$ & \\
\hline Lower third & 41 & $30(73.1)$ & $11(26.8)$ & \\
\hline $\begin{array}{l}\text { Histopathological } \\
\text { grading }\end{array}$ & & & & $0.012^{\mathrm{b}}$ \\
\hline Gx & 11 & $9(81.8)$ & $2(18.2)$ & \\
\hline G1 & 31 & $26(83.9)$ & $5(16.1)$ & \\
\hline G2 & 58 & $37(63.8)$ & $21(36.2)$ & \\
\hline G3 & 39 & $18(46.2)$ & $21(53.8)$ & \\
\hline G4 & 1 & $1(100)$ & $0(0)$ & \\
\hline Depth of invasion & & & & $<0.001^{\mathrm{b}}$ \\
\hline pT0-1 & 59 & $50(84.7)$ & $9(15.3)$ & \\
\hline pT2 & 12 & $10(83.3)$ & $2(16.7)$ & \\
\hline pT3 & 56 & $27(48.2)$ & $29(51.8)$ & \\
\hline pT4 & 13 & $4(30.8)$ & $9(69.2)$ & \\
\hline Regional lymph node & & & & $<0.001^{\mathrm{b}}$ \\
\hline $\mathrm{pNO}$ & 71 & $61(85.9)$ & $10(14.1)$ & \\
\hline $\mathrm{pN} 1$ & 36 & $20(55.6)$ & $16(44.4)$ & \\
\hline $\mathrm{pN} 2$ & 22 & $9(40.9)$ & $13(59.1)$ & \\
\hline $\mathrm{pN} 3$ & 11 & $1(9.1)$ & $10(90.9)$ & \\
\hline Distant metastasis & & & & $1.000^{\mathrm{c}}$ \\
\hline M0 & 138 & $90(64.3)$ & $48(34.3)$ & \\
\hline M1 & 2 & $1(50.0)$ & $1(50.0)$ & \\
\hline pStage & & & & $<0.001^{\mathrm{b}}$ \\
\hline 0-I & 48 & $42(87.5)$ & $6(12.5)$ & \\
\hline II & 31 & $27(87.1)$ & $4(12.9)$ & \\
\hline III & 59 & $21(35.6)$ & $38(64.4)$ & \\
\hline IV & 2 & $1(50.0)$ & $1(50.0)$ & \\
\hline Residual tumor & & & & $<0.001^{\mathrm{c}}$ \\
\hline Negative & 126 & $89(70.6)$ & $37(29.4)$ & \\
\hline Positive & 14 & $2(14.3)$ & $12(85.7)$ & \\
\hline Preoperative CT & & & & $0.034^{\mathrm{b}}$ \\
\hline None & 90 & $63(70.0)$ & $27(30.0)$ & \\
\hline FP & 42 & $26(61.9)$ & $16(38.1)$ & \\
\hline $\mathrm{DCF}$ & 8 & $2(25.0)$ & $6(75.0)$ & \\
\hline Postoperative therapy & & & & $0.002^{b}$ \\
\hline None & 111 & $80(72.0)$ & $31(28.0)$ & \\
\hline CT & 19 & $8(42.1)$ & $11(57.9)$ & \\
\hline Chemoradiotherapy & 10 & $3(30.0)$ & $7(70.0)$ & \\
\hline
\end{tabular}

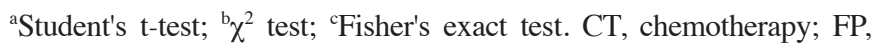
5-fluorouracil and cisplatin; DCF, docetaxel, cisplatin and 5-fluorouracil.
Table II. Primary detectable recurrence site following thoracoscopic esophagectomy.

\begin{tabular}{lc}
\hline Recurrent pattern & Patients, $\mathrm{n}(\%)$ \\
\hline Recurrence & $49(35.0)$ \\
Hematogeneous & $24(17.1)$ \\
Lung & 14 \\
Liver & 8 \\
Kidney & 3 \\
Intestine & 2 \\
Bone & 1 \\
Brain & 1 \\
Adrenal gland & 1 \\
Spleen & 1 \\
Thoracic wall & 1 \\
Lymphatic & $26(18.6)$ \\
Mediastinal regional lymph node & $8(5.7)$ \\
Distant lymph node & $21(15.0)$ \\
Pleural dissemination & $5(3.6)$ \\
Peritoneal dissemination & $2(1.4)$ \\
Local & $4(2.9)$
\end{tabular}

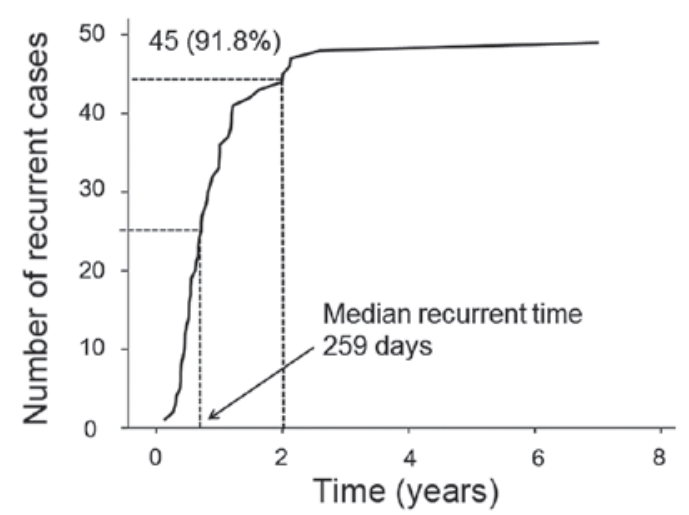

Figure 1. Time to recurrence in the 49 patients who developed recurrence following thoracoscopic esophagectomy.

$5(3.6 \%)$, peritoneal dissemination in $2(1.4 \%)$ and local recurrence in $4(2.9 \%)$ (Table II). Lymphatic recurrence within the mediastinal regional lymphatic stations was identified only in $8(5.7 \%)$ of the 140 patients.

Factors predicting recurrence. Univariate analysis for relapse-free survival showed that a tumor location in the upper third of the esophagus, stage of pT3 or pT4, presence of nodal metastasis, pStage of III or IV, presence of a residual tumor, performance of preoperative chemotherapy and performance of postoperative therapy were statistically significant independent variables (Table III). Relapse-free survival curves according to the depth of tumor invasion, lymph node metastasis status, pathological stage and residual tumor are shown in Fig. 2. Multivariate analysis showed that only nodal metastasis and a positive residual tumor were statistically significant variables for relapse-free survival (Table IV). The correlations 
Table III. Univariate analysis for relapse-free survival.

\begin{tabular}{|c|c|c|}
\hline Variables & $\operatorname{HR}(95 \% \mathrm{CI})$ & P-value \\
\hline \multicolumn{3}{|l|}{ Age } \\
\hline$\geqq 70$ years & $1.072(0.626-1.834)$ & 0.801 \\
\hline \multicolumn{3}{|l|}{ Gender } \\
\hline Male & $0.894(0.465-1.722)$ & 0.738 \\
\hline \multicolumn{3}{|l|}{ Location } \\
\hline Upper third & $1.919(1.051-3.502)$ & $0.034^{\mathrm{a}}$ \\
\hline \multicolumn{3}{|l|}{ Histology } \\
\hline Poorly SCC & $0.818(0.461-1.451)$ & 0.493 \\
\hline \multicolumn{3}{|l|}{ Tumor depth } \\
\hline pT3, pT4 & $2.988(1.729-5.161)$ & $<0.001^{\mathrm{a}}$ \\
\hline \multicolumn{3}{|c|}{ Nodal metastasis } \\
\hline Positive & $4.097(2.304-7.286)$ & $<0.001^{\mathrm{a}}$ \\
\hline \multicolumn{3}{|c|}{ Distant metastasis } \\
\hline Positive & $1.612(0.223-11.655)$ & 0.636 \\
\hline \multicolumn{3}{|l|}{ pStage } \\
\hline III or IV & $3.961(2.299-6.825)$ & $<0.001^{\mathrm{a}}$ \\
\hline \multicolumn{3}{|c|}{ Residual tumor } \\
\hline Positive & $4.393(2.307-8.363)$ & $<0.001^{\mathrm{a}}$ \\
\hline \multicolumn{3}{|c|}{ Preoperative therapy } \\
\hline Performed & $1.700(1.0008-2.865)$ & $0.046^{\mathrm{a}}$ \\
\hline \multicolumn{3}{|c|}{ Postoperative therapy } \\
\hline Performed & $2.384(1.370-4.149)$ & $0.002^{\mathrm{a}}$ \\
\hline
\end{tabular}

${ }^{\mathrm{a}} \mathrm{P}<0.05$. Analysis was performed using the Cox regression model. HR, hazard ratio; CI, confidence interval; SCC, squamous cell carcinoma.

Table IV. Significant variables in multivariate analysis for relapse-free survival.

\begin{tabular}{lcc}
\hline Variables & HR $(95 \%$ CI $)$ & P-value \\
\hline $\begin{array}{l}\text { Nodal metastasis } \\
\text { Positive }\end{array}$ & $3.870(2.170-6.902)$ & $<0.001$ \\
$\begin{array}{l}\text { Residual tumor } \\
\text { Positive }\end{array}$ & $3.807(1.990-7.285)$ & $<0.001$ \\
\hline
\end{tabular}

Analysis was performed using the Cox regression model. The full-model approach incorporating all candidate variables was used. Only significant variables are listed. HR, hazard ratio; CI, confidence interval.

between these variables and recurrence patterns were investigated (Table V). The presence of pathological lymph node metastasis at the primary surgery was associated with hematological, lymphatic and local recurrence. A noncurative surgery with a residual tumor was associated with hematological recurrence, pleural dissemination and local recurrence.

Distribution of lymphatic recurrence. The distribution of lymph node metastasis at the primary surgery and the lymphatic recurrence was also investigated in each lymphatic
A

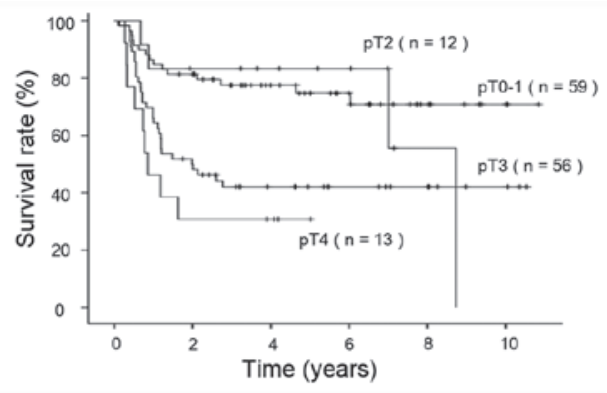

B

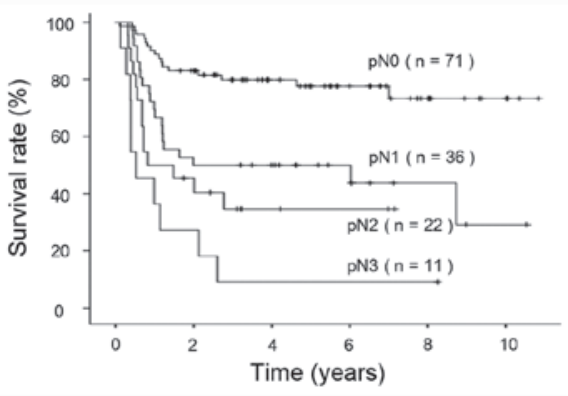

C

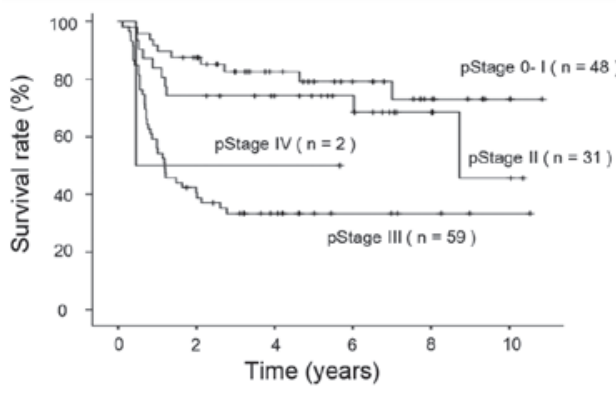

D

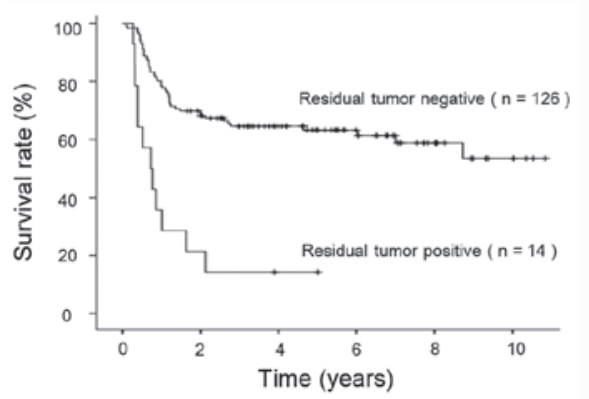

Figure 2. Relapse-free survival curves of patients who underwent thoracoscopic esophagectomy according to (A) tumor depth of invasion ( $\mathrm{pT}$ ), (B) lymph node status ( $\mathrm{pN}$ ), (C) pathological stage (pStage) and (D) presence of a pathological residual tumor. Clinicopathological characteristics (tumor invasion, node, metastasis and stage) were based on the TNM classification (7th edition) by the International Union Against Cancer.

station as classified by Japanese classification of esophageal cancer (Table VI). Metastasis was frequently identified in the upper mediastinal region, including around the bilateral recurrent laryngeal nerves. The right tracheobronchial, pretracheal, abdominal para-aortic pretracheal, posterior mediastinal or abdominal para-aortic stations were not routinely dissected, as these stations were not defined as regional stations. The recurrence rate in each station within the regional nodes was less than the initial metastatic rate. The distribution of lymphatic metastasis and recurrence was compared using McNemar's test to assess the association between initial metastasis and lymphatic recurrence in each patient. The distribution of lymphatic recurrence was the same as the appearance of the 
Table V. Recurrence patterns according to recurrence risk factors.

\begin{tabular}{|c|c|c|c|c|c|c|}
\hline \multirow[b]{3}{*}{ Recurrent pattern } & \multicolumn{6}{|c|}{ Recurrent risk factors } \\
\hline & \multicolumn{3}{|c|}{ Lymph node metastasis } & \multicolumn{3}{|c|}{ Residual tumor } \\
\hline & $\begin{array}{c}\text { Negative, } \mathrm{n}(\%) \\
\mathrm{n}=71\end{array}$ & $\begin{array}{c}\text { Positive, } \mathrm{n}(\%) \\
\mathrm{n}=69\end{array}$ & P-value & $\begin{array}{c}\text { Negative, } n(\%) \\
n=126\end{array}$ & $\begin{array}{c}\text { Positive, } \mathrm{n}(\%) \\
\mathrm{n}=14\end{array}$ & P-value \\
\hline Hematological & $7(9.9)$ & $17(24.6)$ & $0.02^{\mathrm{a}}$ & $18(14.3)$ & $6(43)$ & $0.016^{\mathrm{b}}$ \\
\hline Lymphatic & $3(4.2)$ & $23(33.3)$ & $<0.001^{\mathrm{b}}$ & $22(17.5)$ & $4(28.6)$ & $0.294^{\mathrm{b}}$ \\
\hline Pleural dissemination & $2(2.8)$ & $3(4.3)$ & $0.678^{\mathrm{b}}$ & $2(1.6)$ & $3(21.4)$ & $0.007^{\mathrm{b}}$ \\
\hline Peritoneal dissemination & $0(0)$ & $2(2.9)$ & $0.241^{\mathrm{b}}$ & $2(1.6)$ & $0(0)$ & $1.000^{\mathrm{b}}$ \\
\hline Local & $0(0)$ & $4(5.8)$ & $0.056^{\mathrm{a}}$ & $1(0.8)$ & $3(21.4)$ & $0.003^{b}$ \\
\hline
\end{tabular}

${ }^{\mathrm{a}} \chi^{2}$ test. ${ }^{\mathrm{b}}$ Fisher's exact test.

Table VI. Lymph node metastasis at the primary surgery and lymphatic recurrence in each lymphatic station.

\begin{tabular}{|c|c|c|c|}
\hline Lymphatic station & $\begin{array}{l}\text { Lymph node metastasis at } \\
\text { the primary surgery, } \mathrm{n}(\%)\end{array}$ & $\begin{array}{c}\text { Recurrence with } \\
\text { lymphatic metastasis, n }(\%)\end{array}$ & P-value \\
\hline Cervical & $17(12.1)$ & $9(6.4)$ & 0.077 \\
\hline Mediastinum & $61(43.5)$ & $13(9.3)$ & $<0.001$ \\
\hline Upper mediastinum & $42(30.0)$ & $10(7.1)$ & $<0.001$ \\
\hline Upper thoracic paraesophageal & $7(5.0)$ & $1(0.7)$ & 0.07 \\
\hline Right recurrent laryngeal & $22(15.7)$ & $1(0.7)$ & $<0.001$ \\
\hline Left recurrent laryngeal & $20(14.3)$ & $4(2.9)$ & 0.001 \\
\hline Right tracheobronchial $^{\mathrm{b}}$ & $0(0.0)$ & $1(0.7)$ & - \\
\hline Left tracheobronchial & $7(5.0)$ & $2(1.4)$ & 0.125 \\
\hline Pre-tracheal ${ }^{\mathrm{b}}$ & $2(1.4)$ & $6(4.3)$ & 0.219 \\
\hline Middle mediastinum & $25(17.9)$ & $0(0.0)$ & - \\
\hline Subcarinal & $9(6.4)$ & $0(0.0)$ & - \\
\hline Middle thoracic para-esophageal & $14(10.0)$ & $0(0.0)$ & - \\
\hline Right main bronchus & $9(6.4)$ & $0(0.0)$ & - \\
\hline Left main bronchus & $8(5.7)$ & $0(0.0)$ & - \\
\hline Lower mediastinum & $22(15.7)$ & $3(2.1)$ & $<0.001$ \\
\hline Lower thoracic para-esophageal & $20(14.3)$ & $0(0.0)$ & - \\
\hline Supra-diaphragmatic & $5(3.6)$ & $1(0.7)$ & 0.219 \\
\hline Posterior mediastinal & $0(0.0)$ & $3(2.1)^{\mathrm{c}}$ & - \\
\hline Abdominal & $34(25.0)$ & $10(7.1)$ & $<0.001$ \\
\hline Perigastric & $34(24.3)$ & $2(1.4)$ & $<0.001$ \\
\hline Celiac artery & $6(4.3)$ & $3(2.1)$ & 0.508 \\
\hline Abdominal para-aortic ${ }^{b}$ & $0(0.0)$ & $5(3.6)$ & - \\
\hline
\end{tabular}

${ }^{\mathrm{a}} \mathrm{McNemar}$ 's test; ${ }^{\mathrm{b} l y m p h}$ node station out of regional nodes; ${ }^{\mathrm{c} 3}$ patients developed lymph node metastasis in the dorsal area of the thoracic aorta, where lymphatic dissection was impossible from the right thoracic approach.

initial metastasis in the dissected lymph nodes in the cervical area; however, it was different from that in the mediastinal and abdominal areas. Additionally, the distribution of recurrence differed from that of the initial metastatic site with the exception of the upper thoracic paraesophageal, left tracheobronchial, supradiaphragmatic and celiac artery stations.

\section{Discussion}

The present study evaluated the risk factors that influence the development of recurrence following thoracoscopic radical esophagectomy and showed that nodal metastasis and a positive residual tumor were statistically significant variables 
for relapse-free survival. Lymphatic recurrence within the mediastinum (particularly in the stations around the bilateral recurrent laryngeal nerves where lymphatic metastasis was commonly identified) was infrequent and independent of the initial metastatic distribution.

In general, the reported recurrence rate following curative radical esophagectomy by open thoracotomy is 42 to $52 \%$ (4-7). A previous study reported a recurrence rate of $47 \%$ following thoracoscopic esophagectomy (14). In the present study, a $35.0 \%$ recurrence rate following VATS-esophagectomy, including noncurative surgeries, was observed. The recurrence rate following curative resection was lower at $29.4 \%$. These results appear to be more favorable than those of previous studies (4-7). Our previous study introduced the preoperative instead of postoperative chemotherapy from 2008, as a Japanese randomized clinical trial (JCOG9907) showed the superiority of preoperative compared to postoperative chemotherapy (21). Patients with advanced-stage cancer underwent multimodal therapy. The present univariate analysis showed that the performance of preoperative and/or postoperative therapy was associated with recurrence. These factors were not considered significant variables for relapse-free survival in the multivariate analysis. In February 2012, our department changed the neoadjuvant chemotherapy protocol to a more powerful regimen comprising docetaxel, cisplatin and 5-fluorouracil for stage III and IV tumors. The ability of this regimen to reduce recurrence could not be fully assessed due to the small number of patients and short observation period in the present study. Another method of treating advanced esophageal cancer involves neoadjuvant chemoradiotherapy followed by surgery. The neoadjuvant chemoradiotherapy results in a significant improvement in outcomes, with a recurrence pattern that is different than that associated with surgery alone (22). However, preoperative chemoradiotherapy reportedly limits the available treatments for recurrence and is thus associated with a poor prognosis, as radiotherapy cannot be performed following recurrence (7). Preoperative chemotherapy instead of chemoradiotherapy was performed in the present study, as the main purpose of radiotherapy is local control of the tumor. Favorable locoregional control can be achieved by thoracoscopic surgery without radiotherapy. Radiotherapy can be performed in cases of noncurative surgeries or recurrence. A multicenter, triple-arm randomized controlled trial is now being conducted in Japan (JCOG1109) to determine whether cisplatin +5 -fluorouracil chemotherapy, docetaxel + cisplatin + 5-fluorouracil chemotherapy, or chemoradiation therapy is the most effective preoperative therapy. Further studies are required to fully show the therapeutic effect of preoperative and/or postoperative therapy.

In the present study, T4 tumors that appeared to be resectable following preoperative chemotherapy were included. In these cases, the structure around the tumor bed could not be completely removed to preserve the adjacent organs. Therefore, the surgical curability was only assessed by postoperative histopathological examination. Furthermore, cases were occasionally encountered involving an intraoperative diagnosis of T4, despite such tumors being determined as resectable by preoperative examination. When the tumor was proven to be residual postoperatively, adjuvant chemoradiotherapy around the tumor bed was performed. As it is difficult to accurately determine resectability in the preoperative period, clinically resectable cases were included in the study. Recently, the therapeutic effect of preoperative chemotherapy for T4 tumors increased the chance of resection. Furthermore, the prognosis was favorable when curative resection with negative surgical margins was obtained by the therapeutic effect of preoperative chemotherapy (17).

In the present study, nodal metastasis and a residual tumor were considered statistically significant factors for worse relapse-free survival. These two factors were correlated with multiple recurrence patterns. Lymph node metastasis was proven to be an indicator of hematological, lymphatic and local recurrence. The presence of a residual tumor was correlated with hematological recurrence, pleural dissemination and local recurrence. Despite the performance of postoperative chemoradiotherapy, a residual tumor was a statistically significant predictive factor for recurrence. All the patients with residual tumors accompanied by lymph node metastasis developed postoperative recurrence. However, to the best of our knowledge, 2 of the 4 patients with a residual tumor without lymph node metastasis remain alive without recurrence. Previous studies have shown that a greater depth of invasion and/or lymph node metastasis extension were risk factors for recurrence following radical lymphatic dissection $(3,8,9)$. In the present study, the depth of tumor invasion was not a significant risk factor for relapse-free survival. The results also showed a favorable local effect of thoracoscopic surgery.

The lymphatic recurrence pattern was carefully analyzed in each lymphatic station in association with the distribution of the lymphatic metastasis at the initial surgery, to assess the effect of mediastinal dissection during the thoracoscopic procedure. Lymphatic recurrence was observed in the right tracheobronchial, pretracheal, posterior mediastinal and abdominal para-aortic stations in $1(0.7 \%), 6(4.3 \%), 3(2.1 \%)$ and $5(3.6 \%)$ patients, respectively. Dissection could not be performed by a right thoracic approach in the dorsal thoracic aorta; 3 patients developed postoperative lymphatic recurrence in this region. Other lymphatic stations were defined as nonregional stations and had not been dissected at the primary surgery. Therefore, these recurrences were considered distant lymphatic metastases in this study. Lymphatic recurrence within the mediastinum, particularly in the station around the bilateral recurrent laryngeal nerves, was infrequent and independent of the initial metastatic distribution. However, lymphatic recurrence was encountered around the left recurrent laryngeal nerve in 4 patients. Recurrence was frequent at the border zone between the left recurrent laryngeal and left cervical paraesophageal stations. Thus, this border zone should be completely dissected by an additional cervical approach following thoracoscopic dissection. Conversely, lymphatic recurrence was significantly correlated with the initial metastasis in the cervical and celiac artery regions. Cervical lymphatic recurrence could be managed by additional surgical resection in the majority of cases. However, resection of lymphatic recurrence around the celiac artery is not easy in the presence of a reconstruction conduit around the recurrent nodes or direct invasion of the tumor to the celiac artery. Although our standard abdominal procedure during the primary surgery is hand-assisted laparoscopic surgery, we generally select conventional laparotomy for patients with abdominal metastasis. The feasibility of dissection by laparoscopic surgery has not been proven, even for gastric cancer. Among the mediastinal regional lymphatic stations, lymphatic 
recurrence was associated with the initial lymphatic metastasis in the upper thoracic paraesophageal, left tracheobronchial and supradiaphragmatic stations. Lymphatic recurrence was observed in each station and in 1 patient for each station. During the performance of the thoracoscopic procedure, the right bronchial artery was routinely transected for mediastinal dissection. We attempted to preserve the left bronchial artery to maintain the bronchial circulation during the lymphatic dissection procedure around left tracheobronchial station. The degree of lymphatic dissection is dependent upon the position of the left upper bronchial artery. Not uncommonly, complete lymphatic dissection of this station is impossible. Another problem is lymphatic recurrence in the supradiaphragmatic station. The VATS-esophagectomy procedure was performed with the patient in the left lateral position. Creation of a surgical space in the lower mediastinum is more difficult with the patient in the left lateral position compared to the prone position, in which the effects of gravity could be used to collapse the right lung (23). The use of artificial pneumothorax by insufflation of carbon dioxide to create a wider surgical space was recently introduced.

In conclusion, VATS-esophagectomy with radical lymph node dissection was proven to provide favorable locoregional control in the present study. In particular, mediastinal regional lymphatic recurrence was infrequent and independent of lymph node metastasis at the initial surgery. Nodal metastasis is a significant risk factor for lymphatic and hematological recurrence following a curative surgery. Lymphatic recurrence is likely to develop in the cervical, abdominal celiac artery or abdominal para-aortic lymphatic stations. Although mediastinal regional lymphatic recurrence is infrequent, careful attention must be administered to recurrence at sites without regional nodes, including the pretracheal station and dorsal portion of the thoracic aorta. A residual tumor is a risk factor for local, hematological and pleural metastasis. When pathological curative resection cannot be carried out despite the performance of preoperative chemotherapy, careful follow up is necessary. The inhibitory effect of preoperative chemotherapy on recurrence remains uncertain. Further techniques to reduce recurrence are required.

\section{References}

1. Akiyama H, Tsurumaru M, Udagawa H and Kajiyama Y: Radical lymph node dissection for cancer of the thoracic esophagus. Ann Surg 220: 364-372, 1994.

2. Fujita H, Kakegawa T, Yamana H, Shima I, Toh Y, Tomita Y, Fujii T, Yamasaki K, Higaki K and Noake T: Mortality and morbidity rates, postoperative course, quality of life and prognosis after extended radical lymphadenectomy for esophageal cancer. Comparison of three-field lymphadenectomy with two-field lymphadenectomy. Ann Surg 222: 654-662, 1995.

3. Udagawa H, Ueno M, Shinohara H, Haruta S, Kaida S, Nakagawa $\mathbf{M}$ and Tsurumaru $\mathbf{M}$ : The importance of grouping of lymph node stations and rationale of three-field lymphoadenectomy for thoracic esophageal cancer. J Surg Oncol 106 742-747, 2012.

4. Bhansali MS, Fujita H, Kakegawa T, Yamana H, Ono T, Hikita S, Toh Y, Fujii T, Tou U and Shirouzu K: Pattern of recurrence after extended radical esophagectomy with three-field lymph node dissection for squamous cell carcinoma in the thoracic esophagus. World J Surg 21: 275-281, 1997.

5. Mariette C, Balon JM, Piessen G, Fabre S, Van Seuningen I and Triboulet JP: Pattern of recurrence following complete resection of esophageal carcinoma and factors predictive of recurrent disease. Cancer 97: 1616-1623, 2003.
6. Nakagawa S, Kanda T, Kosugi S, Ohashi M, Suzuki T and Hatakeyama K: Recurrence pattern of squamous cell carcinoma of the thoracic esophagus after extended radical esophagectomy with three-field lymphadenectomy. J Am Coll Surg 198: 205-211, 2004.

7. Miyata H, Yamasaki M, Kurokawa Y, Takiguchi S, Nakajima K, Fujiwara Y, Konishi K, Mori M and Doki Y: Survival factors in patients with recurrence after curative resection of esophageal squamous cell carcinomas. Ann Surg Oncol 18: 3353-3361, 2011.

8. Osugi H, Takemura M, Takada N, Hirohashi K, Kinoshita H and Higashino M: Prognostic factors after oesophagectomy and extended lymphadenectomy for squamous oesophageal cancer. Br J Surg 89: 909-913, 2002.

9. Shimada H, Okazumi S, Matsubara H, Nabeya Y, Shiratori T, Shimizu T, Shuto K, Hayashi H and Ochiai T: Impact of the number and extent of positive lymph nodes in 200 patients with thoracic esophageal squamous cell carcinoma after three-field lymph node dissection. World J Surg 30: 1441-1449, 2006.

10. Cuschieri A, Shimi S and Banting S: Endoscopic oesophagectomy through a right thoracoscopic approach. J R Coll Surg Edinb 37: 7-11, 1992.

11. Nagpal K, Ahmed K, Vats A, Yakoub D, James D, Ashrafian H, Darzi A, Moorthy K and Athanasiou T: Is minimally invasive surgery beneficial in the management of esophageal cancer? A meta-analysis. Surg Endosc 24: 1621-1629, 2010.

12. Biere SS, van Berge Henegouwen MI, Maas KW, Bonavina L, Rosman C, Garcia JR, Gisbertz SS, Klinkenbijl JH, Hollmann MW, de Lange ES, et al: Minimally invasive versus open oesophagectomy for patients with oesophageal cancer: A multicentre, open-label, randomised controlled trial. Lancet 379: 1887-1892, 2012.

13. Osugi H, Takemura M, Higashino M, Takada N, Lee S and Kinoshita H: A comparison of video-assisted thoracoscopic oesophagectomy and radical lymph node dissection for squamous cell cancer of the oesophagus with open operation. Br J Surg 90: 108-113, 2003.

14. Thomson IG, Smithers BM, Gotley DC, Martin I, Thomas JM, O'Rourke P and Barbour AP: Thoracoscopic-assisted esophagectomy for esophageal cancer: Analysis of patterns and prognostic factors for recurrence. Ann Surg 252: 281-291, 2010.

15. Kinjo Y, Kurita N, Nakamura F, Okabe H, Tanaka E, Kataoka Y, Itami A, Sakai Y and Fukuhara S: Effectiveness of combined thoracoscopic-laparoscopic esophagectomy: Comparison of postoperative complications and midterm oncological outcomes in patients with esophageal cancer. Surg Endosc 26: 381-390, 2012.

16. Ninomiya I, Osugi H, Fujimura T, Kayahara M, Takamura H, Takemura M, Lee S, Nakagawara H, Nishimura G and Ohta T: Results of video-assisted thoracoscopic surgery for esophageal cancer during the induction period. Gen Thorac Cardiovasc Surg 56: 119-125, 2008.

17. Ninomiya I, Okamoto K, Fujimura T, Fushida S, Osugi H and Ohta T: Oncologic outcomes of thoracoscopic esophagectomy with extended lymph node dissection: 10-year experience from a single center. World J Surg 38: 120-130, 2014.

18. Sobin LH, Gospodarowicz MK and Wittekind C: TNM classification of malignant tumors. 7th edition. Wiley-Blackwell, Oxford, 2010

19. Japanese Esophageal Society: Japanese Classification of Esophageal Cancer, Tenth Edition: Part I. Esophagus 6: 1-25, 2009.

20. Japanese Esophageal Society: Japanese Classification of Esophageal Cancer, Tenth edition: Parts II and III. Esophagus 6: 71-94, 2009.

21. Ando N, Kato H, Igaki H, Shinoda M, Ozawa S, Shimizu H, Nakamura T, Yabusaki H, Aoyama N, Kurita A, et al: A randomized trial comparing postoperative adjuvant chemotherapy with cisplatin and 5-fluorouracil versus preoperative chemotherapy for localized advanced squamous cell carcinoma of the thoracic esophagus (JCOG9907). Ann Surg Oncol 19: 68-74, 2012.

22. Smit JK, Guler S, Beukema JC, Mul VE, Burgerhof JG, Hospers GA and Plukker JT: Different recurrence pattern after neoadjuvant chemoradiotherapy compared to surgery alone in esophageal cancer patients. Ann Surg Oncol 20: 4008-4015, 2013.

23. Ozawa S, Ito E, Kazuno A, Chino O, Nakui M, Yamamoto S, Shimada $\mathrm{H}$ and Makuuchi $\mathrm{H}$ : Thoracoscopic esophagectomy while in a prone position for esophageal cancer: A preceding anterior approach method. Surg Endosc 27: 40-47, 2013. 